\title{
GLAST Large Area Telescope Multiwavelength Opportunities
}

\author{
David J. Thompson \\ Astroparticle Physics Laboratory, NASA Goddard Space Flight Center, Greenbelt, MD 20771 USA \\ On Behalf of the GLAST LAT Multiwavelength Coordinating Group
}

\begin{abstract}
High-energy gamma-ray sources are inherently nonthermal, multiwavelength objects. With the launch of the Gamma-ray Large Area Space Telescope (GLAST) scheduled for later this year, the GLAST Large Area Telescope (LAT) Collaboration invites cooperative efforts from observers at all wavelengths. Among the many topics where multiwavelength studies will maximize the scientific understanding, two stand out for particular emphasis: (1) Active Galactic Nuclei. The study of AGN gamma-ray jets through time variability and spectral modeling can help link the accretion processes close to the black hole with the large-scale interaction of the AGN with its environment; (2) Unidentified Gamma-ray Sources. New gamma-ray sources need first to be identified with known objects seen at other wavelengths using position, spectrum, or time variability, and then multiwavelength studies can be used to explore the astrophysical implications of high-energy radiation from these sources. Observers interested in any type of coordinated observations should contact the LAT Multiwavelength Coordinating Group.
\end{abstract}

Keywords: gamma rays $\cdot$ multiwavelength $\cdot$ active galactic nuclei $\cdot$ unidentified sources.

PACS: $95.40 .+\mathrm{s} \cdot 95.55 . \mathrm{Ka} \cdot 95.85 . \mathrm{Pw}$

\section{THE VALUE OF MULTIWAVELENGTH STUDIES}

As can be seen in Figure 1, all known classes of high-energy gamma-ray sources are multiwavelength objects, with emission seen across much of the electromagnetic spectrum. Because astrophysical gamma rays are produced by nonthermal processes involving high-energy particles, a broad spectral range is expected.

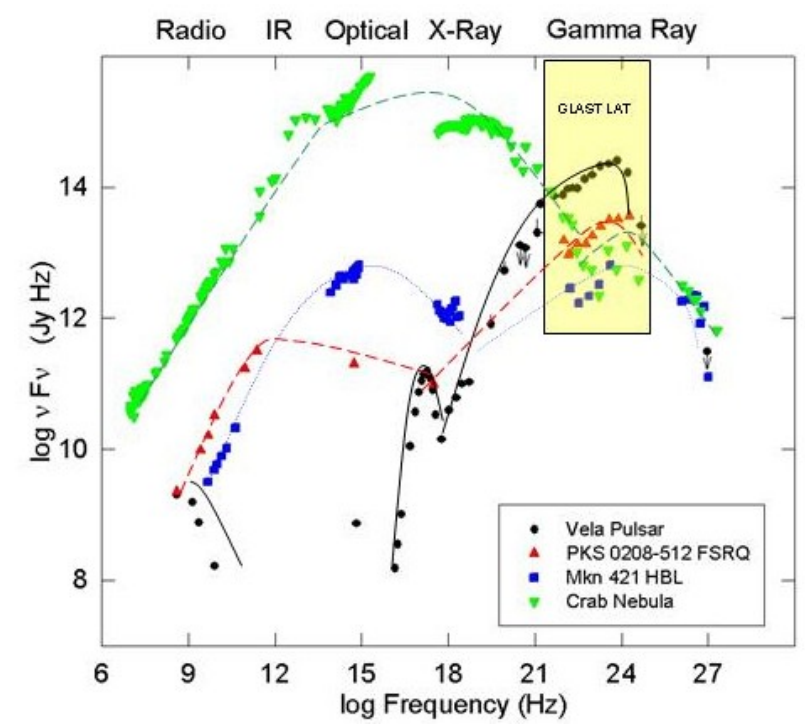

FIGURE 1. Broad-band Spectral Energy Distributions of example gamma-ray sources. Lines are guides for the eye, not fits. 
Understanding such objects is inherently a multiwavelength challenge. A full picture can only be obtained by finding the sources of all the radiation.

For gamma-ray observations, multiwavelength studies are also essential for identifying sources in the first place. With the exception of bright pulsars (recognizable by a measured period and period derivative), gamma-ray observations are unlikely to yield an identification by themselves. Finding counterparts at other wavelengths provides the critical identification information: precision source location, distance, mass and composition, and overall energetics.

\section{LAT TEAM MULTIWAVELENGTH PLANNING}

\section{Programs for Known Source Classes}

The LAT team is working with many observers on planning for studies of known gamma-ray source classes:

- Pulsars: the key is having independent timing information from radio and X-ray observations, since the gamma-ray data are sparse compared to the rotation period of pulsars [1]. Radio and X-ray proposals have been submitted, and some timing programs are underway. More information is always welcome.

- Blazars: these bright, highly-variable sources require a wide range of planning efforts. See also [2].

- Finding new blazars is important, because LAT may detect more blazars than are currently cataloged. Example programs include CGraBS [3], a combination of radio and optical studies, and VLBA Imaging and Polarization Survey (VIPS), a program using radio polarization with optical followup.

- Multiwavelength monitoring is critical, because few patterns of variability have been found. Some example cooperative efforts include MOJAVE [4], RATAN-600 [5], and U. Michigan in the radio, and the Global Telescope Network (GTN), Perugia, and Pan-STARRS in the optical.

- Multiwavelength campaigns of two types are being planned [6]:

1. Target of Opportunity (TOO) campaigns are important for catching flaring sources (e.g. [7]). A critical element is quick communication using a variety of methods.

2. Planned Intensive Campaigns (PIC) mobilize maximum resources by setting up preplanned observations. Several of these have been proposed by the LAT team.

- Gamma-ray Bursts: planning for these involves drawing on the many highly-successful efforts already in place, including Swift, the GCN communications system, and the Interplanetary Network.

\section{Planning for Source Identification}

The GLAST LAT team is developing Figure of Merit approaches for statistical association of unidentified LAT sources with possible new object classes. This process involves many catalogs at various wavelengths, plus parameters such as spectra or variability, e.g. [8]. We will need some specific examples to confirm such associations and move beyond identification toward understanding the sources. Some possibilities (see also [9], [10]) include.

1. “Top-Down” approach: look for an X-ray or $\mathrm{TeV}$ counterpart with better source localization.

2. "Bottom-Up" approach: look for a flat-spectrum radio counterpart that might be a blazar.

3. "Variability/Spectral Modeling" approach: look for consistency across the spectrum.

\section{SCIENTIFIC COMMUNITY INVOLVEMENT}

In terms of involvement in GLAST science, the most important factor to remember is that both GLAST instruments have huge fields of view. With GLAST operated in scanning mode (the default for planning), both the GBM and the LAT will survey the entire sky about every three hours. The consequence is that whatever your favorite source is, GLAST will observe it. Involvement can be determined by the science, not the GLAST observing 
plan. The GBM data (bursts) become public immediately. The LAT data are not generally public during Cycle 1, but there are exceptions, and the LAT team is eager to cooperate with observers with correlative data.

\section{Multiwavelength Blazar Studies}

Here are some suggestions for anyone interested in participating in LAT blazar studies:

Pre-Launch:

- Help identify new blazars. LAT will probably detect more blazars than are currently cataloged.

- Join the GTN or the Whole Earth Blazar Telescope (WEBT) to monitor blazars or participate in campaigns.

- Sign up for the Gamma-ray Multiwavelength e-mail list by contacting David.J.Thompson@nasa.gov

During the First Year (when most LAT data are not public):

- Use the public data from 20 prominent sources (light curves and energy spectra to be posted to the Web) for scientific analysis - most of these are blazars. See http://glast.gsfc.nasa.gov/ssc/data/policy/LAT_Year_1_Data_Release.html

- Bright blazar flare data will be made public, along with the name of a LAT contact who will help coordinate analysis. Join us or use the data on your own. TOO proposals for other observations can be based on these announcements.

\section{Source Identification Planning}

Here are some suggestions for anyone interested in participating in LAT source identification studies:

Pre-Launch:

- Model candidate gamma-ray sources to predict which ones might be observable.

- Start learning about the LAT data system through the GLAST Science Support Center.

During the First Year:

- Bright flare data from unidentified sources will be made public, along with the name of a LAT contact who will help coordinate analysis. Join us or use the data on your own.

- A preliminary list of high-confidence source detections will be released in the middle of the first year. Many are likely to be unidentified, and those can become targets for correlated observations and/or modeling.

\section{Other Resources}

Multiwavelength information, including a link to the LAT Web page for planning, can be found on the GLAST Web site http://glast.gsfc.nasa.gov/science/multi/

Many LAT scientists are active in multiwavelength planning and would be glad to discuss plans. They include: Blazars: Jennifer Carson, Jim Chiang, Stefano Ciprini, Werner Collmar, Luigi Foschini, Benoit Lott, Greg Madejski, Julie McEnery, David Paneque, Rita Sambruna, Gianpiero Tagliaferri, and Gino Tosti. Pulsars: David Smith, Steve Thorsett and Roger Romani. Unidentified or Diffuse Sources: Patrizia Caraveo, Seth Digel, Stefan Funk, Isabelle Grenier, Olaf Reimer, and Kent Wood.

\section{REFERENCES}

1. S. Thorsett, these proceedings 2007.

2. B. Lott, these proceedings 2007.

3. S. Healey, R. Romani, these proceedings 2007.

4. M. L. Lister, these proceedings 2007.

5. L. Fuhrmann, these proceedings 2007.

6. G. Tosti, these proceedings 2007.

7. L. Foschini, these proceedings 2007.

8. V. Lonjou and J. Knödlseder, these proceedings 2007.

9. P. Caraveo and O. Reimer, these proceedings 2007.

10. D. J. Thompson, "The Multiwavelength Approach to Unidentified Gamma-Ray Sources, in Multiwavelength Approach to Unidentified Gamma-Ray Sources, edited by K.S. Cheng and G. E. Romero, Dordrecht: Springer, 2005, pp. 1-7. 